\section{Alkohol erhöht Rhinitisrisiko}

\section{Es gab immer schon den Verdacht, dass ein hoher Alkoholkonsum das Risiko für eine allergische Rhinitis erhöhen könnte. Eine große Kohortenstudie stützt jetzt diese These.}

D änische Forscher dokumentierten die Daten einer Kohorte von 5.870 Frauen im Alter zwischen 20 und 29 Jahren. Zu Beginn, in den Jahren 1991 bis 1993, berichtete keine von ihnen über Rhinitissymptome. Neben anderen potenziellen Risikofaktoren wurde auch der Alkoholkonsum über einen Ernährungsfragebogen erfasst. Im Median tranken die Frauen 3,5 alkoholische Getränke pro Woche - Däninnen sind bekannt für ihren für Frauen relativ hohen Alkoholkonsum. Je mehr Alkohol die Frauen zu sich nahmen, umso häufiger waren sie auch Raucherinnen.

Nach im Median 7,8 Jahren hatten 831 der Frauen (14\%) nach eigenen Angaben eine saisonale allergische Rhinitis entwickelt, 523 (9\%) eine perenniale Rhinitis. Frauen, die regelmäßig mehr als 14 alkoholische Getränke pro Woche zu sich nahmen, gaben zu 78\% häufiger Symptome einer perennialen Rhinitis an als Frauen, die weniger als ein alkoholisches Getränk pro Woche konsumier-

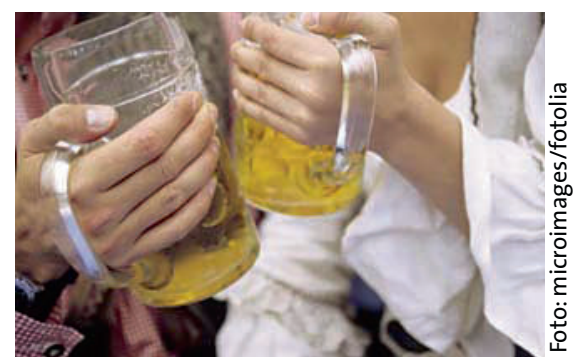

Oans, zwoa, Heischnupf' $n$ ? ten. Der Zusammenhang war dosisabhängig. Keinen Einfluss schien die Höhe des Alkoholkonsums dagegen auf das Risiko für eine saisonale Rhinitis zu haben.

Überraschenderweise konnte kein Zusammenhang zwischen dem Rauchverhalten und dem Rhinitisrisiko hergestellt werden. Die Studie bestätigte aber einen anderen bekannten Risikofaktor: Wenn ein Elternteil unter Asthma litt, hatten die Töchter ein erhöhtes Rhinitisrisiko. Besonders hoch war das Risiko für eine perenniale Rhinitis, wenn zum asthmakranken Elternteil ein hoher Alkoholkonsum der Tochter dazukam.

Fazit: Frauen, die regelmäßig Alkohol trinken, haben ein erhöhtes Risiko, an einer perennialen allergischen Rhinitis zu erkranken.

Bendtsen $\mathrm{P}$ et al. Alcohol consumption and the risk of self-reported perennial and seasonal allergic rhinitis in young adult women in a population-based cohort study. Clin Exp Allergy 2008; 38: 1179-85

\title{
Nasale Lipopolysaccharide triggern Asthma
}

\author{
Bei allergischer Rhinitis ist der Etagenwechsel mit der Entwicklung \\ eines Asthmas ein bekanntes Phänomen. Möglicherweise sind nicht \\ nur Inhalationsallergene, sondern auch Lipopolysaccharide für die \\ Induktion des allergischen Asthma bronchiale mit verantwortlich.
}

ipopolysaccharide (LPS) finden sich Lin hoher Konzentration im Hausstaub. Werden diese Endotoxine gramnegativer Bakterien inhaliert, kann es zu einem lokalen Anstieg von Leukozyten und einer gesteigerten Zytokinausschüttung kommen.

Die Effekte von LPS an der Nasenschleimhaut untersuchte eine schwedische Arbeitsgruppe bei 14 Patienten mit einer Rhinitis aufgrund einer Birkenoder Gräserpollenallergie. Alle waren Nichtraucher, benötigten keine Asthmamedikation und waren zu Beginn der Studie symptomfrei. Die Studienteilnehmer erhielten LPS-Extrakt als Nasenspray. Anschließend wurden ihre subjektiven nasalen Beschwerden erfragt, ebenso erfolgten vor und nach der
Applikation Bestimmungen des nasalen Flusswiderstands, der Leukozyten in der Nasallavage sowie des Stickstoffmonoxid-Gehalts der ausgeatmeten Luft. Drei bis sechs Wochen später wurde die Prozedur wiederholt, allerdings erhielten die Teilnehmer dieses Mal 24 Stunden vor der LPS-Anwendung nasal Birken- oder Gräserpollenextrakte appliziert.

Die Applikation von LPS alleine bewirkte nach sechs Stunden eine Zunahme der Leukozyten in der Nasallavage. Klinische Beschwerden traten jedoch nicht auf. Auch die Rhinomanometrie ergab keine Erhöhung des nasalen Widerstands; der NO-Gehalt der ausgeatmeten Luft blieb konstant.

Die Gabe von Allergenextrakt führte zu vorübergehenden lokalen Beschwer- den wie Obstruktion, Niesreiz oder Rhinorrhoe. Die Applikation von LPS einen Tag später hatte eine Zunahme der Leukozyten in der Lavage um über das Dreifache zur Folge. Nasale Symptomatik und nasaler Flusswiderstand veränderten sich zwar nicht signifikant, die NO-Konzentration der ausgeatmeten Luft stieg allerdings signifikant an. Dies galt nicht nur für die nasale Exspiration, sondern auch für die pulmonale NO-Konzentration.

Fazit: Bei Allergiekranken führt die nasale Applikation von LPS allein nur zum Anstieg der Leukozyten in der Nasallavage. Belastet man die Patienten einen Tag zuvor mit Allergenen, bewirkt die LPS-Applikation auch eine Zunahme der pulmonalen NO-Konzentration. Damit kommen LPS möglicherweise als Triggerfaktor für die Entwicklung eines allergischen Asthmas in Frage.

Bachar $O$ et al. Lipopolysaccharide administration to the allergic nose contributes to lower airway inflammation. Clin Exp Allergy 2007; 37: 1773-80 